\title{
Review of: "Prediction and analysis of EDM performances considering random multiple-pulse discharges based on geometric optimization modeling"
}

\author{
Yanjun $\mathrm{Lu}^{1}$ \\ 1 Shenzhen University
}

Potential competing interests: The author(s) declared that no potential competing interests exist.

This paper presents a new model about EDM technology considering random multiple-pulse discharges. The structure of the paper is clear and reasonable. However, there are many questions need be improved and revised. Especially, the English language of this paper needed to be greatly revised. Some specific concerns/comments are listed below:

1. The title needs to be revised to easily read for readers. The paper needs proofreading to improve the readability and clarity of the paper, especially the abstract, introduction and conclusion. The conclusions were summarized with ambiguity.

2. Generally, in the process of electrical spark discharge, there are both spark discharge and arc discharge, why not consider the arc discharge situation?

3. Some diagrams are not formatted properly. For example, Fig. 2(a) is lack of scale. Fig. 2(b), Fig. 6 and Fig. 12 and so on are lack of a description of abscissa and ordinate. Suggest add some information in Fig. 11. Fig. 16 is not clear.

4. How are the heat affected area and recast layer generated? Please give a more detailed description.

5. What is the correlation between single pulse and multi-pulse discharges? What is its material removal mode? How to control the multi-pulse discharge?

6. Please add photos or topography of EDM machined samples.

7. In the description about Fig. 18, Oxygen is present to a depth of $5.31 \mu \mathrm{m}$, so the actual thickness of the recast layer is $5.31 \mu \mathrm{m}$. Whether this statement is accurate or reliable? Can the recast layer be measured? 
